\title{
Xerostomia and its Dental Implications: A Review
}

\author{
Madhumathi $\mathbf{V}^{1}$, Sowmya $\mathbf{S}^{2}$, Swamy $\mathbf{R}^{3}$
}

\begin{abstract}
Xerostomia is a relatively common complaint often related to the use of certain pharmacological agents. Knowledge about xerostomia, its effect on oral health among the aged population and provision of modified dental care for these patients are the objectives of this review. Further explaining the methods to improve the comfort and oral function of dentate and edentate patients with dry mouth.
\end{abstract}

Keywords: Xerostomia, Artificial saliva, Reservoir.

${ }^{1}$ Senior lecturer
Department of Prosthodontics
KGFCDS, KGF
${ }^{2}$ Senior lecturer
Department of Prosthodontics
JSSDC\&H, A Constituent College of JSS University,
Mysore

${ }^{3}$ Professor
Department of Prosthodontics
JSSDC\&H, A constituent College of JSS University,
Mysore

JSSDC\&H, A constituent College of JSS University,
Mysore

\section{Contact Author}

Dr Madhumathi V madhumathivg@gmail.com

J Oral Health Comm Dent 2013;7(3)166-169

\section{INTRODUCTION}

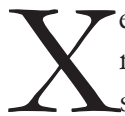
rostomia is defined as dryness of mouth from the lack of normal complaint among the elderly and according to a study approximately $30 \%$ of the population aged 65 and above experience this disorder (1). Individuals with xerostomia complain of dryness of mouth, problems with eating, speaking and swallowing affecting their nutrition as well as psychological health (2,3). It becomes very important to understand problems associated with dry mouth and their management in order to improve the oropharyngeal health and quality of life of the elderly,who constitute significant proportion of the present population.

\section{ETIOLOGY OF XEROSTOMIA}

Drug-induced dry mouth is the most common cause, because the vast majority of older adults are being treated with at least one medication that causes salivary hypofunction (1). Xerostomia is a side effect of a wide variety of medications $(4,5)$ such as antihistaminics, antidepressants, antihypertensives, antianxiety agents, diuretics, antiparkinsonian drugs, antiemetics and bronchodilators. Therapeutic radiation to head and neck, systemic diseases (diabetes mellitus, HIV, emotional stress) and diseases involving the salivary glands (6) are the other possible causes.

\section{EFFECTS OF REDUCED SALIVARY FLOW}

The oral symptoms of xerostomia are

- Difficulty in normal oral and oropharyngeal functions including eating, speaking and swallowing.

- Reduced dilution of plaque acids and antimicrobial protection predisposing to gingivitis and rapidly progressing caries in sites normally spared by the disease, loss of restorations and carious de coronation (2).

- A negative effect on masticatory ability and efficiency, contributing to protein energy malnutrition among elderly denture wearers (7). Extreme discomfort in wearing dentures and dislodgement of dentures at rest is also common. Poor retention is compounded by the reduction in surface tension, rendering the dentures looser and potentially more traumatic.

- The buccal mucosa, tongue and lips tend to stick to the denture predisposing to mucosal abrasion and ulcerations, which can be a major concern for patients (3). The subsequent speech and eating difficulties that may develop can impair social interactions and may cause some patients to avoid social engagements.

- In addition to the increased risk of plaque accumulation, caries, periodontal and mucosal trauma associated with 


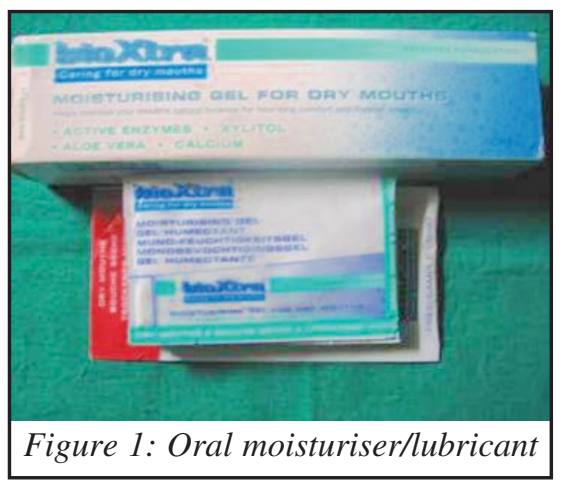

denture use, susceptibility to candidiasis and angular stomatitis may be increased (3).

\section{DIAGNOSIS AND TREATMENT CONSIDERATION}

A variety of treatment options are available to the clinician depending on the cause and severity of the disease. The first step $(8,9)$ in treating patients with xerostomia is establishing a diagnosis. Multidisciplinary approach to the diagnosis and the management of underlying systemic conditions is imperative to reduce oral complications (10). The communication among this team of health care practitioners is critical, because many older people have concomitant medical problems and poly pharmaceutical complications. For the medication induced xerostomia, liaison with a patient's general medical practitioner to discuss the timing, dosage or change in medication may reduce the severity of the problem. It would be appropriate to measure the patients non stimulated salivary flow rates before and after altering their medication in order to gauge the success of treatment.

The second step $(8,9)$ is to schedule frequent dental evaluations to assess patients for oral complications of low salivary output.

\section{Saliva Stimulation and Substitu- tion}

For patients with remaining viable salivary gland tissue, stimulation techniques are helpful. Sugar-free chewing gum, candies and mints can stimulate salivary output. The U.S. Food and Drug Administration has approved two secretagogues (11) pilocarpine and cevimeline, for the treatment
Table 1: Composition of artificial saliva

\begin{tabular}{|ll|}
\hline Components & Quantity \\
\hline Water & $500 \mathrm{ml}$ \\
\hline Xylitol & $20 \mathrm{gm}$ \\
\hline Potassium chloride & $1.2 \mathrm{gm}$ \\
\hline Sodium chloride & $0.843 \mathrm{gm}$ \\
\hline Magnesium chloride & $0.051 \mathrm{gm}$ \\
\hline Tricalcium phosphate & $20 \mathrm{ml}$ \\
\hline Peppermint food flavour & $5 \mathrm{ml}$ \\
\hline Carboxymethylcellulose & $10 \mathrm{gm}$ \\
\hline Sodium hydroxide & $20 \mathrm{ml}$ \\
\hline
\end{tabular}

of Xerostomia (as in irradiated patients or patients with Sjogrens syndrome) and salivary hypo function. These drugs are effective in increasing secretions and diminishing xerostomic complaints in patients with sufficient exocrine tissue. In edentulous patients use of fruit flavoured confectionery may be useful to stimulate residual salivary function (2). Recent evidence indicates that secretion of mucous saliva from the palate improves measurably after drinking $2 \mathrm{~L}$ of water when chewing vigorously or when taking oestrogen or Pilocarpine.

Dry mucosal surfaces and dysphagia are treated with oral moisturizers and lubricants (Figure 1), artificial saliva (Table 1) and night time use of bedside humidifiers (12). Clinicians must instruct patients to drink fluids while eating, particularly if foods are dry and rough.

\section{DENTAL IMPLICATIONS}

Preventive measures such as frequent dental and oral evaluations, good oral hygiene,low-sugar diet, daily use of topical fluorides and antimicrobial mouth rinses are critical to help prevent dental caries (13), periodontal diseases, mucosal infections and other oral complications.

Dentures should be avoided where possible and a shortened dental arch should be accepted when appropriate (2). If unavoidable certain modifications in the denture fabrication procedure and denture designs are recommended for edentulous (partial /complete) patients with dry mouth. It is important to explain, and rehearse all clini- cal procedures with the patient as the discomfort will be kept to a minimum with their co-operation.

\section{Partial Dentures}

A short span edentulous area is best restored by a fixed partial denture (14) as it reduces mucosal contact and further irritation. Fixed partial dentures should have full coverage retainers and easily cleaned pontics and connectors. The margins of retainers should be supragingival.

Removable partial dentures (3) should be designed in such a way that it should be entirely tooth supported with minimum tissue coverage. Denture components should be highly smooth and polished to minimize plaque formation and facilitate soft tissue movement. Connectors should be kept at least $4 \mathrm{~mm}$ clear of the gingival margin to aid in reduction of plaque stagnation. Conventional gingivally approaching clasp should be avoided because they stand away from the tissue and are likely to catch on the cheeks. A modified de van clasp following the end of the flange and a well contoured and adapted half encircling clasp may minimize this problem. A combination approach using an anterior saddle restored with a bridge or implants and posterior saddles with denture will minimize the size of the connector and the number of gingival margins that must be covered.

Reservoirs can be incorporated into partial removable dentures (2). They can be incorporated in the free end saddle areas with tooth bearing portion forming the lids (Figure 2). The lids of the reservoir are retained by magnets and retentive acrylic interlocks.

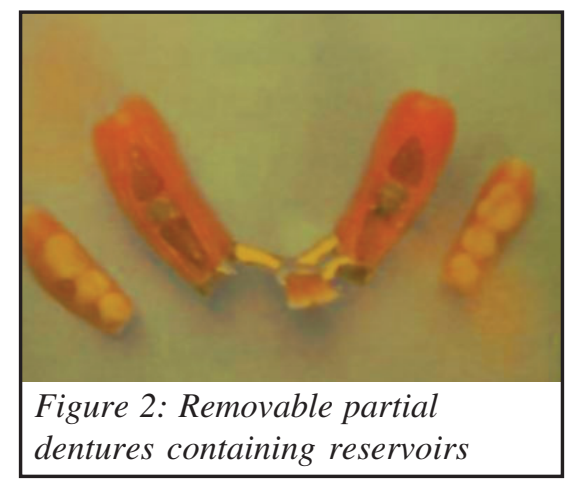




\section{Complete Dentures}

Complete dentures can be very damaging to the dry mouth, so special consideration should be given to clinical and laboratory procedures and aimed at optimizing denture retention and stability.

A careful and gentle approach (3) is essential for patients with dry mouth as the mucosa and lips are friable and easily traumatized. The lips should be coated with petroleum jelly to help with retraction and access to the oral cavity. The operator's gloved fingers should be wetted to prevent them sticking to the soft tissue. A mirror should be used instead of fingers to facilitate insertion of the tray in cases of circumoral scarring due to chronic angular cheilitis or underlying connective tissue diseases. The restricted oral aperture makes access for impression making extremely difficult and painful, so a two part impression may be necessary. Zinc oxide eugenol paste will adhere to the dry mucosa and cause severe irritation/burning sensation. Similarly, impression plaster will adhere to mucosa and abrade it. Therefore such impression materials must be avoided. Silicone impression materials are best tolerated and least traumatic to the mucosa.

Dentures with metal bases exhibit improved accuracy of fit and effective wetting contributing to better retention (15). Interocclusal records are recorded at a closed vertical dimension. Plastic non-anatomic teeth are preferred and are to be placed in the neutral zone. Well finished prosthesis is recommended.

Soft denture liners can be used on the fitting surface of denture routinely to minimize the traumatic forces and patient discomfort.

Retention can be increased by the patient soaking the denture in water, then spraying its entire surface with artificial saliva. The best results are obtained by moistening the mouth with a sip of water and then spraying with the saliva substitute before placing the dentures. A common disadvantage of these moistening techniques is the relative short retention time

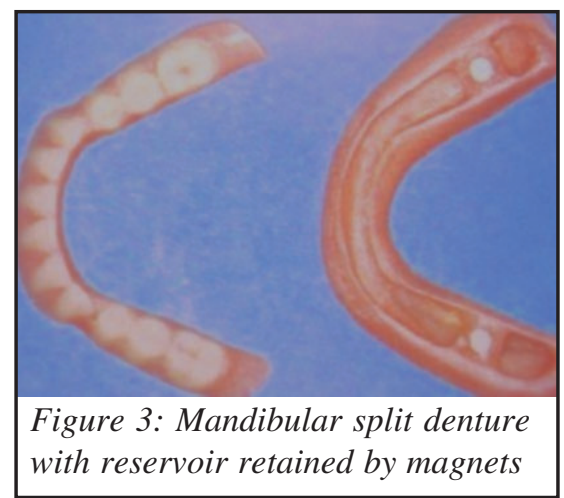

of saliva substitute in the oral cavity requiring reapplication every few hours, which is highly inconvenient for the patients. To circumvent this problem slow release devices like artificial saliva reservoirs within removable prosthesis have been suggested for patients with xerostomia (16-19). A reservoir denture (Figure 3) can hold and release increased volume (between 2 and 4 ml) of artificial saliva.

\section{Reservoir Bite Guards}

Unfortunately the reservoir dentures (3) are expensive and difficult to make and may require the services of specialist laboratory. A simple alternative in the form of bite guards have been suggested, which can be worn by dentate and partially dentate patients or over a conventional complete denture. It is a modified bite guard with bilateral reservoirs. Reservoir bite guards were constructed from double layer of polyvinyl acetate with bilateral inverted pear shaped reservoirs.

\section{Dental Implants}

To date there have been only a few reports on the use of implants in patients with xerostomia. These case reports (20) indicate that these patients can be successfully treated with osseointegrated implants resulting in enhanced patient comfort (14). In these patients placement of implant and their integration into the underlying bone occur normally unless there is a significant immunosuppression or patient under high doses of steroid therapy. Since no clinical trials have been performed with dental implants in xerostomia patients knowledge of patient's susceptibility to peri-implantitis cannot be determined. When implants are considered to be viable therapeutic options, it seems prudent for clinicians to adhere to the following guidelines (21)-

- Prior to implant placement, the underlying cause of the xerostomia should be properly diagnosed and treated.

- Any oral bacterial infections such as periodontitis, caries or fungal infections such as candidiasis should be thoroughly treated prior to implant placement.

- After implant placement, maintenance intervals should be shortened to prevent the development of periimplantitis due to the increased plaque formation in these patients.

\section{Discussion}

Various treatment modalities are available for xerostomia depending on the aetiology as mentioned above. However the treatment or combination of treatments is carefully tailored for each patient in order to restore normal oropharyngeal function and reduce patient. Replacement of teeth by dentures is recommended only in cases where it is an absolute necessity. Removable prostheses are fabricated with special care to avoid any trauma to the dry oral mucosa. At the same time priority should be given to optimize retention and stability of the prostheses by the judicious use of denture adhesives, metallic denture bases,salivary stimulants, saliva substitutes, artificial saliva,lubricating devices like reservoir dentures. The better options of fixed prostheses, implant supported prostheses or fully bone anchored implant prostheses should always be verified. Patients wearing implant supported prostheses exhibit increased comfort, stability and retention when compared to mucosa supported prosthesis (14).

Reservoir dentures (16-19) can be complete or partial removable dentures. Reservoirs can be placed in either maxillary or mandibular dentures. Various designs of reservoirs have been suggested. Reservoirs can be incorporated either into an already existing denture or into a newly fabricated denture. Whenever lubricating devices are planned the nature, size and location of the device particularly the reservoirs are the 
significant criteria to be fulfilled. Maximum space should be gained for reservoir with the existing constraints of reduced vertical dimension, low occlusal plane level or prominent alveolar ridge contour. And the adjustments in the finished prosthesis should be kept to a minimum in order to avoid perforation of the reservoir. In addition the reservoirs should be cleaned and refilled with ease.

In spite of availability of vast majority of treatments, denture patients with xerostomia are more prone for opportunistic oral infections, uncontrolled caries and denture problems emphasizing the need for regular review by the clinicians at short intervals. Furthermore there is a need for the development of long lasting materials that will improve patient comfort;increase ease of chewing,swallowing and speaking; and reduce common oropharyngeal discomfort; and long term studies of the use of implants in these patients (10).

\section{Conclusion}

Dry mouth problems have a clinically significant deleterious impact on oropharyngeal health, making dental management more challenging: Therefore clinicians must be able to diagnose dry mouth conditions in their elderly patients and provide preventive and interventional treatments to reduce the impact of this on the quality of life of the affected. The combination of various treatment modalities appears to be more appropriate in most of these patients.

\section{REFERENCES}

1. Ship JA, Pillemer SR, Baum BJ. Xerostomia and the geriatric patient. JAm Geriatr Soc 2002;50(3):535-43.

2. Helen L Craddock. An aid to the management of xerostomia in the partially dentate patient. Dent Update 2004;31: 302-04.

3. Pankhurst CL, Dunne SM, Rogers JO. Restorative dentistry in the patient with dry mouth:part two: problems and solutions. Dent Update 1996;23(3):11014.

4. Sreebny LM, Schwartz SS. A reference guide to drugs and dry mouth. Gerodontology 1986;5:75-99.

5. Byrne BE. Oral manifestations of systemic agents. In: ADA guide to dental therapeutics. Chicago: ADA Publishing 1998:469-75.

6. Michael D Turner, Jonathan A Ship. Dry mouth and its Effects on the oral health of elderly people. JADA 2007;138:15-20.

7. George A Zarb, Charles L Bolender. Prosthodontic treatment for edentulous patient, complete denture and implant supported prosthesis. 12th edition St. Louis 2003, Mosby publishing

8. Atkinson JC, Wu A. Salivary gland dysfunction: causes, symptoms, treatment. J Am Dent Assoc 1994;125(4):409-16.

9. Fox PC. Management of dry mouth. Dent Clin North Am 1997;41(4):863-76.

10. Michael T, Leila J, Jonathan AS. Hyposalivation, xerostomia and the complete dentures - A systematic review. J Am Dent Assoc 2008;139(2):146-50.

11. Vivino FB, Al-Hashimi I, Khan Z, et al. Pilocarpine tablets for the treatment of dry mouth and dry eye symptoms in patients with Sjögren syndrome: a randomized, placebo-controlled, fixeddose, multicenter trial. P92-01 Study Group. Arch Intern Med 1999; 159(2):174-81.

12. Ship JA. Diagnosing, managing, and preventing salivary gland disorders. Oral Dis 2002;8(2):77-89.

13. Joel JN, Michael TB, Philip CF. Diagnosis and treatment of xerostomia (dry mouth). Odontology 2009;9:76-83.

14. Joseph J Massad, David R Cagna. Removable prosthodontic therapy and xerostomia. Treatment considerations. Dent Today 2002;21(Issue 6),80-87.

15. Hummel SK, Marker VA, Buschang $P$, et al. A pilot study to evaluate different palate materials for maxillary complete dentures with xerostomic patients. $J$ Prosthodont 1999;8:10-17.

16. Covington JS, Slagle WF, Disney AL. Complete denture salivary fluid reservoirs: a novel approach to xerostomia relief. J Dent Res 1985;64:242.

17. Vissink A, Huisman MC, Gravenmade EJ. Construction of an artificial saliva reservoir in an existing maxillary denture. J. Prosthet Dent 1986;56:70-74.

18. Sinclair GF, Frost PM, Walter JD. New design for an artificial saliva reservoir for the mandibular complete denture. $J$ Prosthet Dent 1996;75:276-80.

19. Mendoza AR, Tomlinson MJ. The split denture: A new technique for artificial saliva reservoirs in mandibular dentures. Austr Dent J 2003;48:(3):190-94.

20. Payne AG, Lownie JF, Van Der Linden WJ. Implant supported prostheses in patients with Sjogren's syndrome a clinical report on three patients. Int J Oral Maxillofac implants 1997;12:679-85.

21. Beikler T, Flemming TF. Implants in the medically compromised patient. Crit Rev Oral Biol Med 2003;14(4):305-16. 